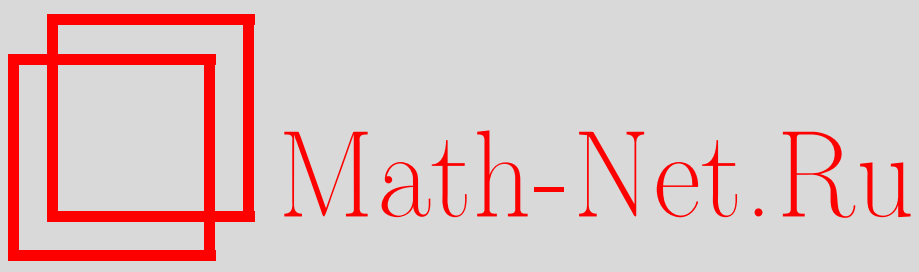

А. А. Талалян, Г. Г. Геворкян, Г. А. Карагулян, О некоторых линейных методах суммирования рядов Фурье, Матем. сб., 1998, том 189, номер 5, 129-152

DOI: https://doi.org/10.4213/sm329

Использование Общероссийского математического портала Math-Net.Ru подразумевает, что вы прочитали и согласны с пользовательским соглашением http: //www. mathnet.ru/rus/agreement

Параметры загрузки:

IP: 54.196 .121 .252

26 апреля 2023 г., $13: 28: 50$ 
УДК 517.51

А.А. Талалян, Г. Г. Геворкян, Г.А. Карагулян

\section{О некоторых линейных методах суммирования рядов Фурье}

В работе даны новые нетрадиционные методы суммирования тригонометрических рядов Фурье от непрерывных функций, обеспечивая их равномерную сходимость.

Библиографиия: 15 названий.

\section{§1. Обозначения и вспомогательные замечания}

Через $C_{2 \pi}$ будем обозначать класс непрерывных на действительной оси $\mathbb{R}=$ $(-\infty,+\infty)$ периодических, с периодом $2 \pi$, функций, а

$$
\begin{aligned}
& D_{n}(x)=\frac{1}{2}+\cos x+\cdots+\cos n x=\frac{\sin \frac{2 n+1}{2} x}{2 \sin x / 2} \\
& F_{n}(x)=\frac{1}{n+1} \sum_{k=0}^{n} D_{k}(x)=\frac{1}{2(n+1)}\left(\frac{\sin \frac{n+1}{2} x}{\sin x / 2}\right)^{2}
\end{aligned}
$$

- ядра Дирихле и Фейера. Обозначим через

$$
S_{n}(f, x)=\frac{a_{0}}{2}+\sum_{k=1}^{n}\left(a_{k} \cos k x+b_{k} \sin k x\right)
$$

частные суммы ряда Фурье интегрируемой на $[-\pi, \pi]$ периодической, с периодом $2 \pi$, функции $f(x)$, т.е.

$$
\begin{aligned}
& a_{k}=a_{k}(f)=\frac{1}{\pi} \int_{-\pi}^{\pi} f(x) \cos k x d x, \quad k=0,1, \ldots, \\
& b_{k}=b_{k}(f)=\frac{1}{\pi} \int_{-\pi}^{\pi} f(x) \sin k x d x, \quad k=1,2, \ldots
\end{aligned}
$$

Хорошо известно, что если $\Lambda=\left\|\lambda_{k}^{(n)}\right\|$ - бесконечная треугольная матрица, где $n=0,1,2, \ldots, k=0,1,2, \ldots, n$, и

$$
u_{n}(f, x, \Lambda)=\frac{a_{0} \lambda_{0}^{(n)}}{2}+\sum_{k=1}^{n} \lambda_{k}^{(n)}\left(a_{k} \cos k x+b_{k} \sin k x\right),
$$

Работа выполнена при финансовой поддержке АО “Закнефтегазпром-Прометей”. 
то для равномерной сходимости последовательности $u_{n}(f, x, \Lambda)$ к $f(x)$ для всех $f(x) \in C_{2 \pi}$ необходимо и достаточно выполнение условий

$$
\begin{gathered}
\lim _{n \rightarrow \infty} \lambda_{k}^{(n)}=1 \quad(k=0,1,2, \ldots) \\
L_{n}(\Lambda)=\int_{-\pi}^{\pi}\left|u_{n}(x, \Lambda)\right| d x=2 \int_{0}^{\pi}\left|u_{n}(x, \Lambda)\right| d x=O(1),
\end{gathered}
$$

где

$$
u_{n}(x, \Lambda)=\frac{1}{\pi}\left(\frac{\lambda_{0}^{(n)}}{2}+\sum_{k=1}^{n} \lambda_{k}^{(n)} \cos k x\right), \quad n=0,1,2, \ldots,
$$

называется ядром линейного оператора $u_{n}(f, x, \Lambda)$. Получению достаточных условий на матрицу $\Lambda$ для вьполнимости соотношения (1.8) при предположении, что (1.7) выполнено, посвящено много глубоких исследований (см. [1]-[4], [13]-[15]). Эффективное достаточное условие для сходимости последовательности $u_{n}(f, x, \Lambda)$ установлено в фундаментальной работе С. М. Никольского [1]:

ТЕорема (С.М. Никольский). Для того чтобъ последовательность $u_{n}(f, x, \Lambda)$ сходиласв равномерно $\kappa f(x)$ для всех $f \in C_{2 \pi}$ необходимо, а в случае, когда $\Delta^{2} \lambda_{k}^{(n)} \leqslant 0\left(k=0,1, \ldots, n-1, \lambda_{n+1}^{(n)}=0, n=0,1, \ldots\right)$ или $\Delta^{2} \lambda_{k}^{(n)} \geqslant 0\left(k=0,1, \ldots, n-1, \lambda_{n+1}^{(n)}=0, n=0,1, \ldots\right)$ также и достаточно, чтобь выполнялись условие (1.7) и следующие условия

$$
\lambda_{k}^{(n)}=O(1)
$$

равномерно относительно всех $n u k$,

$$
\sum_{k=0}^{n} \frac{\lambda_{k}^{(n)}}{n-k+1}=O(1)
$$

əде $\Delta^{2} \lambda_{k}^{(n)}=\lambda_{k}^{(n)}-2 \lambda_{k+1}^{(n)}+\lambda_{k+2}^{(n)}$.

Более того, доказано также, что при выполнении указанных условий на $\lambda_{k}^{(n)}$ средние (1.6), где $f(x)$ - интегрируемая на $[0,2 \pi]$ функция, сходятся к $f(x)$ во всех точках Лебега.

Важньй класс линейных методов суммирования представляют линейные методы, порожденные последовательностями обобщенных мер $\left\{\mu_{n}\right\}$, определенных на $\sigma$-алгебре борелевских множеств действительной оси $\mathbb{R}=(-\infty,+\infty)$ и удовлетворяюших следуюшим четырем условиям:

$$
\begin{gathered}
\lim _{n \rightarrow \infty} \mu_{n}(\mathbb{R})=1, \\
\sup _{n}\left|\mu_{n}\right| \mathbb{R}<+\infty,
\end{gathered}
$$

где $\left|\mu_{n}\right|=\mu_{n}^{+}+\mu_{n}^{-}$, а $\mu_{n}^{+}$и $\mu_{n}^{-}-$положительная и отрицательная части в разложении Хана меры $\mu_{n}$. 
Для любого $A>0$

$$
\lim _{n \rightarrow \infty}\left|\mu_{n}\right|(-\infty,-A)=\lim _{n \rightarrow \infty}\left|\mu_{n}\right|(A,+\infty)=0
$$

Для любого борелевского множества $E \subset(-\infty, 0)$ имеем

$$
\mu_{n}(E)=\mu_{n}(-E), \quad n=1,2, \ldots
$$

где $-E_{n}=\{x:-x \in E\}$.

Обозначив

$$
\lambda_{k}^{(n)}=\int_{-\infty}^{+\infty} \cos k x d \mu_{n}(x), \quad n=1,2, \ldots, \quad 0 \leqslant k \leqslant n
$$

легко видеть, что условие (1.7) для этих $\lambda_{k}^{(n)}$ выполнено.

Легко видеть также, что если обозначить

$$
\begin{gathered}
u_{\mu_{n}}(f, x)=\frac{a_{0} \lambda_{0}^{(n)}}{2}+\sum_{k=1}^{n} \lambda_{k}^{(n)}\left(a_{k} \cos k x+b_{k} \sin k x\right) \\
u_{\mu_{n}}(x)=\frac{1}{\pi}\left(\frac{\lambda_{0}^{(n)}}{2}+\sum_{k=1}^{n} \lambda_{k}^{(n)} \cos k x\right)
\end{gathered}
$$

где $\lambda_{k}^{(n)}$ определены равенствами (1.16), то

$$
\begin{gathered}
u_{\mu_{n}}(x)=\frac{1}{\pi} \int_{\mathbb{R}} D_{n}(x-t) d \mu_{n}(t) \\
u_{\mu_{n}}(f, x)=\int_{-\pi}^{\pi} f(x+t) u_{\mu_{n}}(t) d t=\int_{\mathbb{R}} S_{n}(f, x-t) d \mu_{n}(t) .
\end{gathered}
$$

Из вышеизложенного ясно, что для равномерной сходимости последовательности полиномов $u_{\mu_{n}}(f, x)$ к $f(x)$ на всем классе $C_{2 \pi}$ необходимо и достаточно выполнение условия

$$
\sup _{n} \int_{-\pi}^{\pi}\left|u_{\mu_{n}}(x)\right| d x<+\infty
$$

ибо согласно (1.12)-(1.15) и (1.16) числа $\lambda_{k}^{(n)} \rightarrow 1$ при $n \rightarrow \infty$ длявсех $k=1,2, \ldots$ В обшем же случае, как это следует из (1.13) и (1.19), имеем

$$
\int_{-\pi}^{\pi}\left|u_{\mu_{n}}(x)\right| d x \leqslant \frac{4}{\pi^{2}}\left|\mu_{n}\right|(\mathbb{R}) \ln n+O(1) \leqslant c \ln n+O(1),
$$

где $c=4 / \pi^{2} \sup _{n}\left|\mu_{n}\right|(\mathbb{R})$. 
Если меры $\mu_{n}$ сосредоточены на интервалах $\left(-1 / p_{n}, 1 / p_{n}\right)$ и $\sup _{n} n / p_{n}<+\infty$, то порожденньй ими линейньй метод суммирования регулярный, так как

$$
\begin{aligned}
& \sum_{k=0}^{n-1}\left|\lambda_{k}^{(n)}-\lambda_{k+1}^{(n)}\right|+\left|\lambda_{n}^{(n)}\right| \\
& \quad=\sum_{k=0}^{n-1}\left(\left|\int_{\mathbb{R}} \cos k t d \mu_{n}-\int_{\mathbb{R}} \cos (k+1) t d \mu_{n}\right|\right)+\left|\int_{\mathbb{R}} \cos n t d \mu_{n}\right| \\
& \quad \leqslant \sum_{k=0}^{n-1} \int_{-1 / p_{n}}^{1 / p_{n}} 2|\sin t / 2| d\left|\mu_{n}\right|+\left|\mu_{n}\right|(\mathbb{R}) \\
& \quad \leqslant\left|\mu_{n}\right|(\mathbb{R})+2 n \sin 1 / 2 p_{n}\left|\mu_{n}\right|(\mathbb{R}) \leqslant c<+\infty
\end{aligned}
$$

для всех $n$.

С другой стороны, для любой последовательности $p_{n} \rightarrow+\infty$, для которой последовательность $\left\{n / p_{n}\right\}$ не ограниченная, существует удовлетворяюшая условиям (1.12)-(1.15) последовательность мер $\mu_{n}$, с носителями $\left(-1 / p_{n}, 1 / p_{n}\right)$, для которых соответствующий линейньй метод нерегулярный. В этом можно убедиться, взяв $\lambda_{0}^{(n)}=1, n=1,2, \ldots$, и

$$
\lambda_{k}^{(n)}=\frac{\sin k \cdot 1 / p_{n}}{k \cdot 1 / p_{n}}, \quad 1 \leqslant k \leqslant n, \quad n=1,2, \ldots .
$$

Этот метод порожден последовательностью $\mu_{n}$, которая удовлетворяет условиям (1.12)-(1.15) (см. § 2 настоящей статьи), но, как легко видеть,

$$
\sup _{n}\left(\sum_{k=0}^{n-1}\left|\frac{\sin k \cdot 1 / p_{n}}{k \cdot 1 / p_{n}}-\frac{\sin (k+1) 1 / p_{n}}{(k+1) 1 / p_{n}}\right|+\left|\frac{\sin n \cdot 1 / p_{n}}{n \cdot 1 / p_{n}}\right|\right)=+\infty .
$$

Однако, как легко видеть, на классе рядов Фурье, сходящихся равномерно или в метриках $L_{p}, 1 \leqslant p<+\infty$, на $[0,2 \pi]$, все методы, порожденные последовательностями мер $\mu_{n}$, удовлетворяющих условиям (1.12)-(1.15), регулярны. Точнее, из сходимости последовательности $S_{n}(f, x)$ к $f(x)$ равномерно или в метриках $L_{p}[0,2 \pi], p \geqslant 1$, следует соответственно равномерная сходимость или сходимость в метриках $L_{p}[0,2 \pi], p \geqslant 1$, последовательности $u_{\mu_{n}}(f, x)$ к той же функции $f(x)$.

Было бы интересно выяснить "регулярны" ли эти методы на классе почти всюду сходящихся рядов Фурье? В частности, сходятся ли почти всюду полиномы $u_{\mu_{n}}(f, x)$ для всех $f \in C_{2 \pi}$ ? Заметим также, что в рассматриваемый класс линейных методов суммирования входят многие хорошо известные методы. В частности, ядра Бернштейна

$$
R_{n}(x)=\frac{1}{2}\left[D_{n}\left(x-\gamma_{n}\right)-D_{n}\left(x+\gamma_{n}\right)\right]
$$

представляются в виде

$$
R_{n}(x)=\int_{R} D_{n}(x-t) d \mu_{n}(t)
$$

где дискретная мера $\mu_{n}$ сосредоточена в точках $-\gamma_{n}, \gamma_{n}$ и на каждой из них равна $1 / 2$. 
Методы суммирования с ядрами (1.23), представляюшие обобшения методов Бернштейна-Рогозинского, введены и обстоятельно исследованы Б. С. Стечкиным [9]. Для оценки норм ядер (1.18) во многих случаях применяется равенство (см. [1])

$$
\begin{aligned}
u_{\mu_{n}}(x) & =\frac{1}{\pi}\left(\frac{\lambda_{0}^{(n)}}{2}+\sum_{k=1}^{n} \lambda_{k}^{(n)} \cos k x\right) \\
& =\frac{1}{\pi}\left(\sum_{k=0}^{n-2}(k+1) F_{k}(x) \Delta^{2} \lambda_{k}^{(n)}+\left(\lambda_{n-1}^{(n)}-\lambda_{n}^{(n)}\right) n F_{n-1}(x)+\lambda_{n}^{(n)} D_{n}(x)\right) .
\end{aligned}
$$

Пусть $g(t)$ - определенная на $\mathbb{R}$ функция ограниченной вариации, которая непрерывна слева в каждой точке и

$$
\lim _{t \rightarrow-\infty} g(t)=0, \quad \lim _{t \rightarrow+\infty} g(t)=1 .
$$

Если кроме того $g(t)$ такова, что порожденная ею мера $\mu(t)$ обладает свойством

$$
\mu([a, b))=g(b)-g(a)=\mu((-b,-a])=g(-a+0)-g(-b+0)
$$

для всех $[a, b) \subset(-\infty, 0)$, то как легко видеть, если $\left\{P_{n}\right\}$ - монотонно стремящаяся к $+\infty$ последовательность положительных чисел и если через $\mu_{P_{n}}$ обозначена мера, порожденная функцией $g\left(P_{n} x\right)$, то последовательность мер $\left\{\mu_{P_{n}}\right\}$ будет обладать свойствами (1.12)-(1.15).

Обозначим

$$
u_{\mu_{P_{n}}}(f, x)=u_{g, P_{n}}(f, x), \quad u_{\mu_{P_{n}}}(x)=u_{g, P_{n}}(x) .
$$

Согласно приведенньм вьше обозначениям имеем

$$
\begin{gathered}
u_{g, P_{n}}=\frac{1}{\pi} \int_{\mathbb{R}} D_{n}(x-t) d g\left(P_{n} t\right)=\frac{1}{\pi}\left(\frac{1}{2}+\sum_{k=1}^{n} \lambda_{k}^{(n)} \cos k x\right), \\
u_{g, P_{n}}(f, x)=\int_{\mathbb{R}} S_{n}(f, x-t) d g\left(P_{n} t\right)=\int_{-\pi}^{\pi} f(x+t) u_{g, P_{n}}(t) d t \\
=\frac{a_{0}}{2}+\sum_{k=1}^{n} \lambda_{k}^{(n)}\left(a_{k} \cos k x+b_{k} \sin k x\right),
\end{gathered}
$$

в которых интегралы на $\mathbb{R}$ понимаются как несобственные интегралы Стилтьеса. Далее, учитывая четность меры $\mu_{P_{n}}$, можно обозначить

$$
\begin{aligned}
\lambda_{k}^{(n)} & =\int_{\mathbb{R}} \cos k t d \mu_{P_{n}}(t)=\int_{-\infty}^{+\infty} \cos k t d g\left(P_{n} t\right) \\
& =\int_{-\infty}^{+\infty} \cos \frac{k}{P_{n}} t d g(t)=\widehat{g}\left(\frac{k}{P_{n}}\right)
\end{aligned}
$$

где $\widehat{g}(x)$ - преобразование Фурье-Стилтьеса функции $g(x)$. 
Заметим также, что согласно (1.26) $\lambda_{0}^{(n)}=1$.

Основная часть настояшей статьи посвящена исследованию операторов $u_{g, P_{n}}(f, x)$ для некоторых конкретных $g(x)$ (см. $\left.\S 2\right)$, здесь же мы докажем две простые обшие теоремы, которые будут использованы в дальнейшем.

Обозначим для краткости

$$
\begin{aligned}
L_{n}\left(g, P_{n}\right) & =\int_{-\pi}^{\pi}\left|u_{g, P_{n}}(x)\right| d x=2 \int_{0}^{\pi}\left|u_{g, P_{n}}(x)\right| d x \\
& =\frac{2}{\pi} \int_{0}^{\pi}\left|\frac{1}{2}+\sum_{k=1}^{n} \lambda_{k}^{(n)} \cos k x\right| d x
\end{aligned}
$$

где $\lambda_{k}^{(n)}$ определены равенствами (1.31).

ТЕОРема 1.1. Пусть последовательность мер $\left\{\mu_{n}\right\}$ удовлетворяет условиям (1.12)-(1.15), причем для некоторой последовательности $P_{n}$ положительных чисел, где

$$
\sup _{n} \frac{n}{P_{n}}<+\infty
$$

выполняются равенства

$$
\left|\mu_{n}\right|\left(\left(-\infty,-1 / P_{n}\right)\right)=\left|\mu_{n}\right|\left(\left(1 / P_{n},+\infty\right)\right)=0, \quad n=1,2, \ldots
$$

Тогда

$$
\int_{-\pi}^{\pi}\left|u_{\mu_{n}}(x)\right| d x=\left|\int_{\mathbb{R}} \cos n t d \mu_{n}(t)\right| \cdot \frac{4}{\pi^{2}} \ln n+O(1)
$$

ДокАЗАТЕЛЬство. Имеем

$$
\begin{aligned}
\left|\Delta^{2} \lambda_{k}^{(n)}\right| & =\left|\int_{\mathbb{R}}(\cos k t-2 \cos (k+1) t+\cos (k+2) t) d \mu_{n}(t)\right| \\
\leqslant & \int_{-1 / P_{n}}^{1 / P_{n}} 4 \sin ^{2} \frac{t}{2} d\left|\mu_{n}\right| \leqslant \frac{\left|\mu_{n}\right|(\mathbb{R})}{P_{n}^{2}} \leqslant \frac{c}{P_{n}^{2}} \\
\left|\lambda_{n-1}^{(n)}-\lambda_{n}^{(n)}\right| & =\left|\int_{\mathbb{R}}(\cos (n-1) t-\cos n t) d \mu_{n}\right| \\
& \leqslant \int_{-1 / P_{n}}^{1 / P_{n}} 2\left|\sin \frac{t}{2}\right| d\left|\mu_{n}\right| \leqslant \frac{\left|\mu_{n}\right|(\mathbb{R})}{P_{n}} \leqslant \frac{c}{P_{n}}
\end{aligned}
$$

где $c=\sup _{n}\left|\mu_{n}\right|(\mathbb{R})<+\infty$. Из (1.35), (1.36) и из равенства (1.25), учитывая (1.33), получаем

$$
\begin{aligned}
\int_{-\pi}^{\pi}\left|u_{\mu_{n}}(x)\right| d x & \leqslant\left|\lambda_{n}^{(n)}\right| \frac{1}{\pi} \int_{-\pi}^{\pi}\left|D_{n}(x)\right| d x+\frac{c}{P_{n}^{2}} \sum_{k=0}^{n-2}(k+1)+\frac{c n}{P_{n}} \\
& \leqslant\left|\int_{\mathbb{R}} \cos n t d \mu_{n}(t)\right| \cdot \frac{4}{\pi^{2}} \ln n+O(1) .
\end{aligned}
$$

Обратное неравенство легко следует из (1.25), (1.35) и (1.36). Из доказанной теоремы следует, что при выполнении условий (1.12)-(1.15), (1.33) и (1.34) для 
равномерной сходимости последовательности полиномов $u_{\mu_{n}}(f, x)$ к $f(x)$ для всех $f \in C_{2 \pi}$ необходимо и достаточно, чтобы

$$
\left|\int_{-\infty}^{+\infty} \cos n x d \mu_{n}\right|=O\left(\frac{1}{\ln n}\right)
$$

В частности, если

$$
\frac{1}{P_{n}}=\frac{\pi}{2 n}+O\left(\frac{1}{n \ln n}\right)
$$

то рассматривая меры $\mu_{n}$, сосредоточенные в точках $1 / P_{n},-1 / P_{n}$ и равные $1 / 2$ в каждой из них, получим, что последовательность

$$
\frac{1}{2}\left(S_{n}\left(f, x-1 / P_{n}\right)+S_{n}\left(f, x+1 / P_{n}\right)\right)
$$

равномерно сходится к $f(x)$ для всех $f \in C_{2 \pi}$.

Теорема 1.2. Пусть $g(t)$ имеет ограниченную вариацию на $\mathbb{R}$ и удовлетворяет условиям (1.26) и (1.27), а ее преобразование Фурье-Стилтьеса $\widehat{g}(x)$ дважды дифференцируемо и удовлетворяет условиям

$$
\left|(\widehat{g}(x))^{\prime}\right| \leqslant \frac{c_{g}}{(1+|x|)^{1+\varepsilon}}, \quad\left|(\widehat{g}(x))^{\prime \prime}\right| \leqslant \frac{c_{g}}{(1+|x|)^{2+\varepsilon}}, \quad \varepsilon>0,
$$

где $c_{g}$ - постоянная, зависящая только от $g$. Тогда для любой последовательности $P_{n} \rightarrow+\infty$ выполняется неравенство

$$
L\left(g, P_{n}\right)=\frac{4}{\pi^{2}}\left|\widehat{g}\left(\frac{n}{P_{n}}\right)\right| \ln n+O(1) .
$$

ДокАЗАТЕЛЬСТво. Из (1.32), (1.31) и (1.25) имеем

$$
\begin{aligned}
L\left(g, P_{n}\right) \leqslant & \frac{2}{\pi} \sum_{k=0}^{n-2}\left|\lambda_{k}^{(n)}-2 \lambda_{k+1}^{(n)}-\lambda_{k+2}^{(n)}\right|(k+1) \int_{0}^{\pi} F_{k}(x) d x \\
& +\frac{2}{\pi}\left|\lambda_{n-1}^{(n)}-\lambda_{n}^{(n)}\right| \cdot n \int_{0}^{\pi} F_{n-1}(x) d x+\frac{2}{\pi}\left|\lambda_{n}^{(n)}\right| \int_{0}^{\pi}\left|D_{n}(x)\right| d x \\
= & \sum_{k=0}^{n-2}\left|\widehat{g}\left(\frac{k}{P_{n}}\right)-2 \widehat{g}\left(\frac{k+1}{P_{n}}\right)+\widehat{g}\left(\frac{k+2}{P_{n}}\right)\right| \cdot(k+1) \\
& +\left|\widehat{g}\left(\frac{n-1}{P_{n}}\right)-\widehat{g}\left(\frac{n}{P_{n}}\right)\right| \cdot n+\left|\widehat{g}\left(\frac{n}{P_{n}}\right)\right|\left(\frac{4}{\pi^{2}} \ln n+3\right) \\
= & I_{1}\left(g, P_{n}\right)+I_{2}\left(g, P_{n}\right)+I_{3}\left(g, P_{n}\right) .
\end{aligned}
$$

Так как

$$
\begin{gathered}
\left|\widehat{g}\left(\frac{k}{P_{n}}\right)-2 \widehat{g}\left(\frac{k+1}{P_{n}}\right)+\widehat{g}\left(\frac{k+2}{P_{n}}\right)\right| \leqslant\left|\widehat{g}^{\prime \prime}\left(\xi_{k}^{(n)}\right)\right| \cdot \frac{2}{P_{n}^{2}}, \\
\frac{k}{P_{n}} \leqslant \xi_{k}^{(n)} \leqslant \frac{k+2}{P_{n}}
\end{gathered}
$$


получаем неравенство

$$
\begin{aligned}
I_{1}\left(g, P_{n}\right) & \leqslant \sum_{k=0}^{n-2}\left|\hat{g}^{\prime \prime}\left(\xi_{k}^{(n)}\right)\right| \cdot \frac{k+1}{P_{n}^{2}} \leqslant 2 c_{g} \sum_{k=0}^{n-2} \frac{k+1}{\left(1+k / P_{n}\right)^{2+\varepsilon} P_{n}^{2}} \\
& \leqslant 2 c_{g} \sum_{k=1}^{n-1} \frac{k}{\left(1+(k-1) / P_{n}\right)^{2+\varepsilon} P_{n}^{2}}=2 c_{g} \sum_{k=1}^{n-1} \frac{k P_{n}^{\varepsilon}}{\left(P_{n}+k-1\right)^{2+\varepsilon}} \\
& \leqslant 2 c_{g}\left(\sum_{k=1}^{\left[P_{n}\right]} \frac{k P_{n}^{\varepsilon}}{P_{n}^{2+\varepsilon}}+\sum_{k=\left[P_{n}\right]+1}^{n-1} \frac{P_{n}^{\varepsilon}}{k^{1+\varepsilon}}\right)
\end{aligned}
$$

которое имеет место, начиная с тех номеров $n$, для которых $P_{n} \geqslant 1$.

Из (1.46) следует, что

$$
I_{1}\left(g, P_{n}\right)=O(1) .
$$

Далее, используя первое условие (1.41), можно писать

$$
I_{2}\left(g, P_{n}\right)=n\left|\widehat{g}\left(\frac{n-1}{P_{n}}\right)-\widehat{g}\left(\frac{n}{P_{n}}\right)\right|=\frac{n}{P_{n}}\left|\widehat{g}^{\prime}\left(\frac{n}{P_{n}}-\frac{\theta_{n}}{P_{n}}\right)\right|, \quad 0 \leqslant \theta_{n} \leqslant 1 .
$$

Из (1.48) и (1.41) следует

$$
I_{2}\left(g, P_{n}\right) \leqslant 2\left|\left(\frac{n}{P_{n}}-\frac{\theta_{n}}{P_{n}}\right) \cdot \widehat{g}^{\prime}\left(\frac{n}{P_{n}}-\frac{\theta_{n}}{P_{n}}\right)\right| \leqslant 2 \sup _{0 \leqslant t \leqslant n / P_{n}}\left|t \widehat{g}^{\prime}(t)\right|=O(1) .
$$

Так как $\widehat{g}(x)$ - ограниченная функция на $\mathbb{R}$, то

$$
I_{3}\left(g, P_{n}\right)=\left|\widehat{g}\left(\frac{n}{P_{n}}\right)\right|\left(\frac{4}{\pi^{2}} \ln n+3\right)=\frac{4}{\pi^{2}} \widehat{g}\left(\frac{n}{P_{n}}\right) \ln n+O(1) .
$$

Из (1.49), (1.47) и (1.43) следует

$$
L\left(g, P_{n}\right) \leqslant \frac{4}{\pi^{2}} \widehat{g}\left(\frac{n}{P_{n}}\right) \ln n+O(1) .
$$

С другой стороны, из (1.25) и (1.29) следует

$$
L\left(g, P_{n}\right) \geqslant \frac{4}{\pi^{2}} \widehat{g}\left(\frac{n}{P_{n}}\right) \ln n+O(1),
$$

что вместе с (1.51) влечет (1.42) и теорема доказана.

Рассмотрим теперь тот случай, когда

$$
g(x)=\int_{-\infty}^{x} \Phi(t) d t,
$$

где $\Phi(x)$ - интегрируемая на $\mathbb{R}$ четная функция и

$$
\int_{\mathbb{R}} \Phi(x) d x=1 .
$$


В этом случае обозначим

$$
\begin{aligned}
u_{g, P_{n}}(f, x)= & \Phi_{n, P_{n}}(f, x), \quad u_{g, P_{n}}(x)=u_{\Phi, P_{n}}(x), \\
& L_{n}\left(g, P_{n}\right)=L_{n}\left(\Phi, P_{n}\right) .
\end{aligned}
$$

Тогда согласно $(1.29),(1.30)$ и (1.31) будем иметь

$$
\begin{aligned}
u_{\Phi, P_{n}}(x)=\frac{1}{\pi} \int_{\mathbb{R}} D_{n}(x-t) P_{n} \Phi\left(P_{n} t\right) d t=\frac{1}{\pi}\left(\frac{1}{2}+\sum_{k=1}^{n} \lambda_{k}^{(n)} \cos k x\right), \\
L_{n}\left(\Phi, P_{n}\right)=\int_{-\pi}^{\pi}\left|u_{\Phi, P_{n}}(x)\right| d x \\
=\frac{1}{\pi} \int_{-\pi}^{\pi}\left|\int_{\mathbb{R}} D_{n}(x-t) P_{n} \Phi_{n}\left(P_{n} t\right) d t\right| d x \\
\Phi_{n, P_{n}}(f, x)=\int_{\mathbb{R}} S_{n}(f, x-t) P_{n} \Phi\left(P_{n} t\right) d t \\
=\frac{1}{\pi} \int_{-\pi}^{\pi} f(x+t) u_{\Phi, P_{n}}(t) d t \\
=\frac{a_{0}}{2}+\sum_{k=1}^{n} \lambda_{k}^{(n)}\left(a_{k} \cos k x+b_{k} \sin k x\right),
\end{aligned}
$$

где

$$
\lambda_{0}^{(n)}=1, \quad \lambda_{k}^{(n)}=\int_{\mathbb{R}} \cos k t P_{n} \Phi\left(P_{n} t\right) d t=\int_{\mathbb{R}} \cos \frac{k}{P_{n}} t \Phi(t) d t=\widehat{\Phi}\left(\frac{k}{P_{n}}\right) .
$$

Здесь $\widehat{\Phi}(x)$ - преобразование Фурье функции $\Phi$.

Из теоремы 1.1 следует

Теорема 1.3. Пусть $\Phi(x)$ - четная финитная функиия с носителем, лежсащем на отрезке $[-A, A], A>0$. Тогда для любой монотонно стремящейся $\kappa+\infty$ последовательности $P_{n}$, удовлетворяющей условию (1.33), имеет место равенство

$$
L_{n}\left(\Phi, P_{n}\right)=\left|\int_{\mathbb{R}} \cos n t P_{n} \Phi\left(P_{n} t\right) d t\right| \frac{4}{\pi^{2}} \ln n+O(1)=\widehat{\Phi}\left(\frac{n}{P_{n}}\right) \frac{4}{\pi^{2}} \ln n+O(1)
$$

и при $P_{n}=n$ последовательность $\Phi_{n, P_{n}}(f, x)=\Phi_{n, n}(f, x)$ равномерно стремится $\kappa f(x)$ для всех $f \in C_{2 \pi}$ тогда и только тогда, когда

$$
\widehat{\Phi}(1)=\int_{\mathbb{R}} \cos t \Phi(t) d t=0 .
$$

При выполнении жсе условия (1.62) верна оченка

$L_{n}(\Phi, n) \leqslant \int_{\mathbb{R}}|\Phi(t)| d t \cdot\left(2 \sin ^{2} \frac{A}{2 n} \cdot \frac{n(n-1)}{2}+2\left|\sin \frac{A}{2 n}\right| \cdot n\right) \leqslant A\left(\frac{A}{4}+1\right) \int_{\mathbb{R}}|\Phi(t)| d t$. 
ДокАЗАТЕЛЬСтво. Нуждается в проверке только вторая часть утверждения теоремы. Во первых заметим, что

$$
\int_{\mathbb{R}} n \Phi(n t) \cos n t d t=\int_{\mathbb{R}} \Phi(t) \cos t d t=\widehat{\Phi}(1) .
$$

Пользуясь условием (1.62) и неравенствами $(1.35),(1.36)$ и $(1.38)$, где меры $\mu_{n}(E)$ определены равенством

$$
\mu_{n}(E)=\int_{E} n \Phi(n t) d t
$$

а вместо $P_{n}$ взяты числа $n / A$, мы получим неравенство (1.63).

Для применения удобна также следуюшая теорема, представляющая частный случай теоремы 1.2 .

Теорема 1.4. Пусть $\Phi(x)$ - четная интегрируемая на $\mathbb{R}$ функция, удовлетворяющая условию (1.54), а ее преобразование Фурье $\widehat{\Phi}(x)$ дважды дифференцируемо и выполнены условия

$$
\left|(\widehat{\Phi}(x))^{\prime}\right|<\frac{C_{\Phi}}{1+|x|}, \quad\left|(\widehat{\Phi}(x))^{\prime \prime}\right|<\frac{C_{\Phi}}{(1+|x|)^{2+\varepsilon}}, \quad \varepsilon>0
$$

әде $C_{\Phi}-$ постоянная, зависящая только от $\Phi$.

Тогда для любой последовательности $\left\{P_{n}\right\}$ полохительных чисел, монотонно стремящейся $\kappa+\infty$, имеет место равенство

$$
L\left(\Phi, P_{n}\right)=\left|\widehat{\Phi}\left(\frac{n}{P_{n}}\right)\right| \ln n+O(1) .
$$

Отметим, что здесь условие на $(\widehat{\Phi}(x))^{\prime}$ в $(1.66)$ на самом деле следует из условия на $(\widehat{\Phi}(x))^{\prime \prime}$. То же самое верно относительно условий (1.41) в теореме 1.2. В этом нетрудно убедиться, пользуясь тем, что $\widehat{g}(x)$ и $\widehat{\Phi}(x)$ - ограниченные на $\mathbb{R}$ функции.

Доказанные теоремы 1.1-1.4 полезны тем, что условиям этих теорем удовлетворяют многие известные методы суммирования и кроме того, взяв в их формулировках конкретные функции $g(x), \Phi(x)$ можно построить в явном виде различные методы суммирования, имеющие ограниченные постоянные Лебега $L_{n}(g, n)$, $L_{n}(\Phi, n)$. Например, взяв в теореме 1.3

и, полагая

$$
\Phi(x)= \begin{cases}1 / 4|\cos x|, & x \in(-\pi, \pi), \\ 0, & x \notin(-\pi, \pi),\end{cases}
$$

получим

$$
\lambda_{k}^{(n)}=\frac{1}{4} \int_{\mathbb{R}} \cos k x \cdot|\cos n x| d x, \quad n=1,2, \ldots, \quad k=0,1, \ldots, n,
$$

$$
\lambda_{n}^{(n)}=0, \quad \lambda_{0}^{(n)}=1, \quad \lambda_{k}^{(n)}=\frac{\cos k \pi / 2 n}{1-(k / n)^{2}}\left(1-\frac{k}{n} \sin k \pi / 2 n\right), 1 \leqslant k<n .
$$

Согласно второй части формулировки теоремы 1.3 последовательность полиномов

$$
\frac{a_{0}}{2}+\sum_{k=1}^{n} \lambda_{k}^{(n)}\left(a_{k} \cos k x+b_{k} \sin k x\right)=\Phi_{n, n}(f, x)
$$

равномерно сходится к $f(x)$ для всех $f \in C_{2 \pi}$ и при этом

$$
\left|L_{n}(\Phi, n)\right| \leqslant \pi\left(\frac{\pi}{4}+1\right)<2 \pi, \quad n=1,2, \ldots .
$$

Из теоремы 1.4 следует 
ТЕОРема 1.5. Если четная интегрируемая на $\mathbb{R}$ функиия удовлетворяет условиям (1.54) и (1.66), то для любой последовательности $P_{n} \uparrow+\infty, P_{n}>0$, удовлетворяющей условию

$$
P_{n}=O\left(n|\ln n|^{-1 / \varepsilon}\right) \quad(\varepsilon \geqslant 0),
$$

последовательность полиномов $\Phi_{n, P_{n}}(f, x)$ равномерно сходится $\kappa f(x)$ для всех $f \in C_{2 \pi}$.

Действительно, так как $\widehat{\Phi}(x) \rightarrow 0$ при $x \rightarrow \infty$, имеем

$$
-\widehat{\Phi}(x)=\int_{x}^{\infty}(\widehat{\Phi}(t))^{\prime} d t, \quad x>0, \quad \widehat{\Phi}(x)=\int_{-\infty}^{x}(\widehat{\Phi}(t))^{\prime} d t, \quad x<0 .
$$

Поэтому из (1.66) следует

$$
\widehat{\Phi}(x)=O\left(|x|^{-\varepsilon}\right) .
$$

Отсюда из неравенства (1.73) и из равенства (1.67) получим

$$
L\left(\Phi, P_{n}\right)=O(1)
$$

ЗАмечАниЕ. Так как в теоремах 1.3 и $1.4 \widehat{\Phi}(x) \rightarrow 1$ при $x \rightarrow 0$, то из равенств (1.67) и (1.61) следует, что для любой финитной функции $\Phi$ или функции $\Phi$, удовлетворяюшей условию (1.66), сушествует зависящая от $\Phi$ постоянная $C_{\Phi}$ такая, что при $P_{n} \leqslant C_{\Phi} n, n=1,2, \ldots$, последовательность $L\left(\Phi, P_{n}\right)$ стремится к $+\infty$ при $n \rightarrow \infty$. Тогда согласно известной теореме для такой последовательности $\left\{P_{n}\right\}$ сушествует $f \in C_{2 \pi}$ такая, что последовательность полиномов $\Phi_{n, P_{n}}(f, x)$ в некоторой точке $x$ неограниченно расходится.

$\mathrm{B} \S 2$ подробно изучается поведение средних $\Phi_{n, P_{n}}(f, x)$ для некоторых конкретных $\Phi(x)$.

\section{§2. Средние Стеклова частных сумм рядов Фурье}

Пусть $h>0$ и положим

$$
\begin{aligned}
\Phi_{h}^{(1)}(t)=\Phi_{h}(t) & = \begin{cases}1 /(2 h), & t \in[-h, h] \\
0, & t \notin[-h, h]\end{cases} \\
\Phi_{h}^{(m)}(x)=\left(\Phi_{h}^{(m-1)} * \Phi_{h}^{(1)}\right)(x) & =\int_{\mathbb{R}} \Phi_{h}^{(m-1)}(x-t) \Phi_{h}^{(1)}(t) d t, \quad m \geqslant 2 .
\end{aligned}
$$

Нетрудно убедится (см. [5, т. I, с. 264]), что $\Phi_{h}^{(m)}$ - четные положительные функции с носителями $[-m h, m h]$, причем

$$
\int_{\mathbb{R}} \Phi_{h}^{(m)}(t) d t=\int_{-m h}^{m h} \Phi_{h}^{(m)}(t) d t=1
$$

а из (2.1) и (2.2) следует

$$
\widehat{\Phi}_{h}^{(m)}(x)=\int_{-\infty}^{\infty} \cos x t \Phi_{h}^{(m)}(t) d t=\left(\frac{\sin h x}{h x}\right)^{m} .
$$


Обозначим

$$
\Phi_{h, n, P_{n}}^{(m)}(f, x)=\int_{\mathbb{R}} S_{n}(f, x-t) P_{n} \Phi_{h}^{(m)}\left(P_{n} t\right) d t
$$

Тогда в силу четности $\Phi_{h}^{(m)}(t)$ и в силу $(2.4)$ получаем

$$
\Phi_{h, n, P_{n}}^{(m)}(f, x)=\frac{a_{0}}{2}+\sum_{k=1}^{n}\left(\frac{\sin k \cdot h / P_{n}}{k \cdot h / P_{n}}\right)^{m}\left(a_{k} \cos k x+b_{k} \sin k x\right) .
$$

Таким образом, полиномы (2.6) представляют средние Стеклова (порядка $m$ и с шагом $\left.h / P_{n}\right)$ частной суммы $S_{n}(f, x)$.

При $m=1$ полиномы (2.6) согласно (2.1) и (2.5) имеют вид

$$
\Phi_{h, n, P_{n}}^{(1)}(f, x)=\frac{P_{n}}{2 h} \int_{-h / P_{n}}^{h / P_{n}} S_{n}(f, x-t) d t .
$$

Из (2.4) и теоремы 1.3 немедленно вытекает следующая

ТЕОРема 2.1. Для того чтобы полиномы

$$
\begin{aligned}
\Phi_{h, n, n}^{(m)}(f, x) & =n \int_{\mathbb{R}} S_{n}(f, x-t) \Phi_{h}^{(m)}(n t) d t \\
& =\frac{a_{0}}{2}+\sum_{k=1}^{n}\left(\frac{\sin k \cdot h / n}{k \cdot h / n}\right)^{m}\left(a_{k} \cos k x+b_{k} \sin k x\right)
\end{aligned}
$$

равномерно сходились $\kappa f(x)$ для всех $f \in C_{2 \pi}$ необходимо и достаточно, чтобьи $h / \pi$ било чельмм числом.

Таким образом, для любых натуральных $p$ и $m$ и для любой функции $f \in C_{2 \pi}$ полиномы

$$
\frac{a_{0}}{2}+\sum_{k=1}^{n}\left(\frac{\sin \frac{\pi k}{n} p}{\frac{\pi k}{n} p}\right)^{m}\left(a_{k} \cos k x+b_{k} \sin k x\right)
$$

где $a_{k}, b_{k}-$ коэффиициенты Фурье функции $f$, равномерно сходятся к $f(x)$ при $n \rightarrow \infty$.

Это утверждение следует из теоремы 2.1, если в ней полагать $h=p \pi$.

Так как носитель функции $\Phi_{\pi P}^{(m)}(x)$ лежит в интервале $(-\pi P m, \pi P m)$, то пользуясь теоремой 1.3 (см. неравенство (1.63)), получаем следующую оценку сверху констант Лебега $L_{n}\left(\Phi_{\pi P}^{(m)}(x), n\right)$ ядер метода суммирования $(2.6)$

$$
L_{n}\left(\Phi_{\pi, P, n}^{(m)}\right) \leqslant m P \pi\left(\frac{m P \pi}{4}+1\right)
$$

Так как при $m \geqslant 3$ из (2.4) вытекает

$$
\left(\widehat{\Phi}_{h}^{(m)}(x)\right)^{\prime \prime} \leqslant \frac{c}{1+|x|^{2+\varepsilon}}, \quad\left(\widehat{\Phi}_{h}^{(m)}(x)\right)^{\prime} \leqslant \frac{c}{1+|x|^{1+\varepsilon}}
$$

то согласно теореме 1.4 имеет место 
TEOPEMA 2.2. Если $m \geqslant 3 u \Phi(x)=\Phi_{\pi}^{(m)}(x)$, то для любой последовательности $P_{n} \uparrow+\infty$

$$
L_{n}\left(\Phi, P_{n}\right)=\frac{4}{\pi^{2}}\left(\frac{\sin \pi n / P_{n}}{\pi n / P_{n}}\right)^{m} \ln n+O(1)
$$

Из теоремы 2.2 следует

TеОрема 2.3. Если $m \geqslant 3$ и $P_{n}=O\left(n(\ln n)^{-1 / m}\right)$, то для любой функции $f \in C_{2 \pi}$ последовательность полиномов

$$
\Phi_{\pi, n, P_{n}}^{(m)}(f, x)=\frac{a_{0}}{2}+\sum_{k=1}^{n}\left(\frac{\sin k \cdot \pi / P_{n}}{k \cdot \pi / P_{n}}\right)^{m}\left(a_{k} \cos k x+b_{k} \sin k x\right),
$$

где $a_{k}, b_{k}$ - коэффициенты Фурье функиии $f$, равномерно сходится $\kappa f(x)$.

Равенство (2.12) дает необходимое и достаточное условие на $P_{n}$, при котором константы Лебега операторов $\Phi_{\pi, n, P_{n}}^{(m)}(f, x), m \geqslant 3$, равномерно ограничены. Тем самым получено необходимое и достаточное условие на $P_{n}$, при котором $\Phi_{\pi, n, P_{n}}^{(m)}(f, x), m \geqslant 3$, равномерно сходится к $f(x)$ для всех $f \in C_{2 \pi}$. Это условие выражается равенством

$$
\left(\frac{P_{n}}{n}\right)^{m}\left|\sin \frac{\pi n}{P_{n}}\right|^{m} \ln n=O(1) .
$$

Получение такого условия в случае $m=1$ и $m=2$ требует более детального изучения соответствующих ядер преобразований.

В случае $m=1$ имеем

$$
\Phi(t)=\Phi_{\pi}^{(1)}(t)= \begin{cases}1 /(2 \pi), & t \in[-\pi, \pi], \\ 0, & t \notin[-\pi, \pi],\end{cases}
$$

и тогда согласно (1.57) и (1.59) получаем

$$
\begin{aligned}
\Phi_{n, P_{n}}(f, x) & =\int_{\mathbb{R}} S_{n}(f, x-t) P_{n} \Phi\left(P_{n} t\right) d t \\
& =\int_{-\pi}^{\pi} f(x+t) u_{\Phi, P_{n}}(t) d t=\int_{-\pi}^{\pi} f(\tau) F_{n, P_{n}}(x-\tau) d \tau,
\end{aligned}
$$

где обозначено

$$
\begin{aligned}
F_{n, P_{n}}(x)=u_{\Phi, P_{n}}(x) & =\frac{1}{\pi} \int_{\mathbb{R}} D_{n}(x-t) P_{n} \Phi\left(P_{n} t\right) d t \\
& =\frac{P_{n}}{2 \pi^{2}} \int_{-\pi / P_{n}}^{\pi / P_{n}} D_{n}(x-t) d t .
\end{aligned}
$$

Константы Лебега $L_{n}\left(\Phi, P_{n}\right)$ имеют вид

$$
L_{n}\left(\Phi, P_{n}\right)=\int_{-\pi}^{\pi}\left|F_{n, P_{n}}(x)\right| d x=\frac{P_{n}}{2 \pi^{2}} \int_{-\pi}^{\pi}\left|\int_{-\pi / P_{n}}^{\pi / P_{n}} D_{n}(x-t) d t\right| d x .
$$


Согласно теореме 1.3 и равенству (2.4), в котором положено $m=1$ и $h=\pi$, если

$$
\sup _{n} \frac{n}{P_{n}}<+\infty
$$

то

$$
L_{n}\left(\Phi, P_{n}\right)=\widehat{\Phi}\left(\frac{n}{P_{n}}\right) \frac{4}{\pi^{2}} \ln n+O(1)=\frac{4}{\pi^{2}}\left|\frac{\sin \pi \cdot n / P_{n}}{\pi \cdot n / P_{n}}\right| \ln n+O(1) .
$$

Из (2.20) следует, что если

$$
\liminf _{n \rightarrow \infty} \frac{n}{P_{n}}<1
$$

то

$$
\limsup _{n \rightarrow \infty} L_{n}\left(\Phi, P_{n}\right)=+\infty
$$

и тогда последовательность

$$
\Phi_{n, P_{n}}(f, x)=\frac{a_{0}}{2}+\sum_{k=1}^{n}\left(\frac{\sin k \cdot \pi / P_{n}}{k \cdot \pi / P_{n}}\right)\left(a_{k} \cos k x+b_{k} \sin k x\right)
$$

не может равномерно сходится к $f(x)$ для всех $f \in C_{2 \pi}$. Ниже проводим исследование ядер $F_{n, P_{n}}(x)$ в случае, когда $P_{n}=O(n)$. Прежде всего отметим, что $F_{n, P_{n}}(x)$ - четная периодическая функция с периодом $2 \pi$ и

$$
\int_{-\pi}^{\pi} F_{n, P_{n}}(x) d x=1
$$

Далее, имеем

$$
\begin{aligned}
F_{n, P_{n}}(x) & =\frac{P_{n}}{2 \pi^{2}} \int_{-\pi / P_{n}}^{\pi / P_{n}} \frac{\sin (n+1 / 2)(x-t)}{2 \sin (x-t) / 2} d t \\
& =\frac{P_{n}}{2 \pi^{2}} \int_{-\pi / P_{n}}^{\pi / P_{n}} \frac{\sin (x-t)}{2 \operatorname{tg}(x-t) / 2} d t+\frac{P_{n}}{4 \pi^{2}} \int_{-\pi / P_{n}}^{\pi / P_{n}} \cos n(x-t) d t .
\end{aligned}
$$

Откуда следует

$$
F_{n, P_{n}}(x)=\frac{P_{n}}{2 \pi^{2}} \int_{-\pi / P_{n}}^{\pi / P_{n}} \frac{\sin (x-t)}{2 \operatorname{tg}(x-t) / 2} d t+\frac{P_{n}}{4 \pi^{2}} \frac{\sin n \pi / P_{n} \cos x}{n}
$$

Из (2.25) следует

$$
\left|F_{n, P_{n}}(x)\right| \leqslant \frac{P_{n}}{2 \pi^{2}}\left|\int_{-\pi / P_{n}}^{\pi / P_{n}} \frac{\sin n(x-t)}{2 \operatorname{tg}(x-t) / 2} d t\right|+c_{1} \frac{P_{n}}{n}\left|\sin n \pi / P_{n}\right| .
$$

Так как для любого интервала $(a, b)$ имеем

$$
\left|\int_{a}^{b} D_{n}(t) d t\right| \leqslant 4 \pi
$$


то из (2.27) следует

$$
\left|F_{n, P_{n}}(x)\right| \leqslant \frac{2 P_{n}}{\pi}, \quad|x| \leqslant \pi
$$

Теперь оценим второе слагаемое правой части (2.26). Имеем

$$
\begin{aligned}
& \int_{-\pi / P_{n}}^{\pi / P_{n}} \frac{\sin n(x-t)}{2 \operatorname{tg}(x-t) / 2} d t \\
& =\frac{1}{2} \int_{-\pi / P_{n}}^{\pi / P_{n}} \sin n(x-t)\left(\frac{1}{\operatorname{tg}(x-t) / 2}-\frac{1}{(x-t) / 2}\right) d t+\int_{-\pi / P_{n}}^{\pi / P_{n}} \frac{\sin n(x-t)}{x-t} d t .
\end{aligned}
$$

При $P_{n}>2$, что имеет место начиная с некоторого $N$, если $|x| \leqslant \pi$ и $|t| \leqslant \pi / P_{n}$, имеем

$$
-\frac{3}{4} \pi \leqslant \frac{x-t}{2} \leqslant \frac{3}{4} \pi
$$

Так как функция

$$
Q(u)=\frac{1}{\operatorname{tg} u}-u
$$

непрерывно дифференцируема на отрезке $[-3 / 4 \pi, 3 / 4 \pi]$, то к первому слагаемому правой части (2.29) можно применить формулу интегрирования по частям. Получим

$$
\begin{aligned}
& \left|\frac{1}{2} \int_{-\pi / P_{n}}^{\pi / P_{n}} \sin n(x-t)\left(\frac{1}{\operatorname{tg}(x-t) / 2}-\frac{1}{(x-t) / 2}\right) d t\right| \\
& \quad=\left|\int_{x / 2-\pi / 2 P_{n}}^{x / 2+\pi / P_{n}} \sin 2 n u Q(u) d u\right|=\left|-\frac{1}{2 n} \int_{x / 2-\pi / 2 P_{n}}^{x / 2+\pi / P_{n}} Q(u) d \cos 2 n u\right| \\
& \leqslant\left|Q\left(x / 2+\pi / 2 P_{n}\right) \cos n\left(x+\pi / P_{n}\right)-Q\left(x / 2-\pi / 2 P_{n}\right) \cos n\left(x-\pi / P_{n}\right)\right| \cdot \frac{1}{2 n} \\
& \quad+\left|\frac{1}{2 n} \int_{x / 2-\pi / 2 P_{n}}^{x / 2+\pi / P_{n}} \cos 2 n u Q^{\prime}(u) d u\right| .
\end{aligned}
$$

Так как

$$
\begin{gathered}
Q\left(x / 2+\pi / 2 P_{n}\right)=Q(x / 2)+Q^{\prime}\left(\xi_{1}\right) \frac{\pi}{2 P_{n}}, \quad \xi_{1} \in\left(\frac{x}{2}, \frac{x}{2}+\frac{\pi}{2 P_{n}}\right), \\
-Q\left(x / 2-\pi / 2 P_{n}\right)=-Q(x / 2)+Q^{\prime}\left(\xi_{2}\right) \frac{\pi}{2 P_{n}}, \quad \xi_{2} \in\left(\frac{x}{2}-\frac{\pi}{2 P_{n}}, \frac{x}{2}\right),
\end{gathered}
$$

TO

$$
\begin{aligned}
& \left|Q\left(x / 2+\pi / 2 P_{n}\right) \cos n\left(x+\pi / P_{n}\right)-Q\left(x / 2-\pi / 2 P_{n}\right) \cos n\left(x-\pi / P_{n}\right)\right| \\
& \quad \leqslant|Q(x / 2)|\left|\cos n\left(x+\pi / P_{n}\right)-\cos n\left(x-\pi / P_{n}\right)\right|+\frac{\pi}{P_{n}} \max _{\xi \in(-3 / 4 \pi, 3 / 4 \pi)}\left|Q^{\prime}(\xi)\right| \\
& \quad \leqslant c_{2}\left|\sin n \pi / P_{n}\right|+c_{3} \pi / P_{n},
\end{aligned}
$$

где $c_{2}$ и $c_{3}$ - абсолютные постоянные. Из (2.32) и (2.35) следует

$$
\left|\frac{1}{2} \int_{-\pi / P_{n}}^{\pi / P_{n}} \sin n(x-t)\left(\frac{1}{\operatorname{tg}(x-t) / 2}-\frac{1}{(x-t) / 2}\right) d t\right| \leqslant c_{2} \frac{1}{n}\left|\sin \frac{n \pi}{P_{n}}\right|+\frac{c_{3}}{n P_{n}} .
$$


Таким образом, из (2.26), (2.29) и (2.36) получим

$$
\left|F_{n, P_{n}}(x)\right| \leqslant c_{1} \frac{P_{n}}{n}\left|\sin \frac{\pi n}{P_{n}}\right|+\frac{c_{2}}{n}+\frac{P_{n}}{2 \pi^{2}}\left|\int_{-\pi / P_{n}}^{\pi / P_{n}} \frac{\sin n(x-t)}{x-t} d t\right|
$$

для всех $x$, где $c_{1}$ и $c_{2}-$ абсолютные постоянные. Теперь оценим последнее слагаемое правой части (2.37) при $|x|>2 \pi / P_{n}$. Тогда при $t \in\left(-\pi / P_{n}, \pi / P_{n}\right)$ имеем

$$
\left|x^{2}-x t\right|>x^{2}-|x t|>\frac{x^{2}}{2}
$$

и поэтому

$$
\begin{aligned}
& \left|P_{n} \int_{-\pi / P_{n}}^{\pi / P_{n}} \frac{\sin (x-t)}{x-t} d t\right| \\
& \quad \leqslant\left|P_{n} \int_{-\pi / P_{n}}^{\pi / P_{n}}\left(\frac{\sin n(x-t)}{x-t}-\frac{\sin n(x-t)}{x}\right) d t\right|+\left|P_{n} \int_{-\pi / P_{n}}^{\pi / P_{n}} \frac{\sin (x-t)}{x} d t\right| \\
& \quad \leqslant 2 P_{n} \int_{-\pi / P_{n}}^{\pi / P_{n}} \frac{|t|}{x^{2}} d t+\frac{P_{n} \sin n \pi / P_{n}}{n|x|}
\end{aligned}
$$

Из (2.37) и (2.39) получаем

$$
\left|F_{n, P_{n}}(x)\right| \leqslant c_{1} \frac{P_{n}}{n}\left|\sin \frac{\pi n}{P_{n}}\right|+\frac{c_{2}}{n}+\frac{c_{3}}{P_{n} x^{2}}+\frac{c_{4} P_{n}\left|\sin n \pi / P_{n}\right|}{n|x|},
$$

которое имеет место для всех $|x|>2 \pi / P_{n}$. Учитьвая неравенства (2.40) и (2.28), получим

$$
\begin{aligned}
\int_{-\pi}^{\pi} & \left|F_{n, P_{n}}(x)\right| d x \\
& \leqslant \int_{-2 \pi / P_{n}}^{2 \pi / P_{n}}\left|F_{n, P_{n}}(x)\right| d x \\
& +\int_{|x|>2 \pi / P_{n}}\left(\frac{c_{1} P_{n}}{n}\left|\sin \frac{\pi n}{P_{n}}\right|+\frac{c_{2}}{n}+\frac{c_{3}}{P_{n} x^{2}}+\frac{c_{4} P_{n}}{|x|} \frac{\left|\sin \pi n / P_{n}\right|}{n}\right) d x \\
& \leqslant c_{1}+\frac{c_{2} P_{n}}{n}\left|\sin \frac{\pi n}{P_{n}}\right|+c_{3} \ln P_{n}\left|\sin \frac{\pi n}{P_{n}}\right| \cdot \frac{P_{n}}{n}
\end{aligned}
$$

где $c_{1}, c_{2}, c_{3}$ - абсолютные постоянные. Таким образом, (2.41) означает, что

$$
L_{n}\left(\Phi, P_{n}\right)=\int_{-\pi}^{\pi}\left|F_{n, P_{n}}(x)\right| d x \leqslant c \frac{P_{n} \ln P_{n}}{n}\left|\sin \pi n / P_{n}\right| .
$$

Аналогичньм образом можно доказывать обратное неравенство. Таким образом, имеем

$$
c_{2} \frac{P_{n} \ln P_{n}}{n}\left|\sin \pi n / P_{n}\right| \leqslant L_{n}\left(\Phi, P_{n}\right) \leqslant c_{1} \frac{P_{n} \ln P_{n}}{n}\left|\sin \pi n / P_{n}\right| .
$$

Из неравенства (2.42) вытекает следуюшая 
ТЕОРемА 2.4. Последовательность средних

$$
\begin{aligned}
\Phi_{n, P_{n}}(f, x) & =\frac{P_{n}}{2 \pi} \int_{-\pi / P_{n}}^{\pi / P_{n}} S_{n}(f, x-t) d t \\
& =\frac{a_{0}}{2}+\sum_{k=1}^{n}\left(\frac{\sin k \pi / P_{n}}{k \pi / P_{n}}\right)\left(a_{k} \cos k x+b_{k} \sin k x\right),
\end{aligned}
$$

где $P_{n} \rightarrow \infty$, равномерно сходится $\kappa f(x)$ для всех $f \in C_{2 \pi}$ тогда и только тогда, когда

$$
\sup _{n}\left(\frac{P_{n} \ln P_{n}}{n}\left|\sin \frac{\pi n}{P_{n}}\right|\right)<+\infty .
$$

Отсюда вытекает, что каждое из перечисленных ниже условий на $\left\{P_{n}\right\}$ достаточно, чтобы полиномы (2.43) сходились равномерно к $f(x)$ для всех $f \in C_{2 \pi}$, когда

$$
\begin{gathered}
P_{n} \leqslant c \frac{n}{\ln n}, \\
n\left(1-\frac{1}{\ln n}\right) \leqslant P_{n} \leqslant n\left(1+\frac{1}{\ln n}\right)
\end{gathered}
$$

и, наконец, ког да $n / P_{n}-$ натуральное число.

Если же, например,

$$
1<\sup _{n} \frac{n}{P_{n}}<+\infty \text { и } \limsup _{n \rightarrow \infty}\left\{\frac{n}{P_{n}}\right\}>0,
$$

где $\{q\}$ означает расстояние от числа $q$ до ближайшего целого, то (2.46) не выполняется и средние (2.43) для некоторых $f \in C_{2 \pi}$ расходятся.

Условие (2.44) означает, что равномерная сходимость средних $(2.43) \mathrm{k} f(x)$ для всех $f \in C_{2 \pi}$ зависит как от быстроты роста последовательности $\left\{P_{n}\right\}$, так и от арифметических свойств чисел $P_{n}$. Например, при $P_{n}=2 n / 3$ соответствующие средние

$$
\Phi_{n, 2 n / 3}(f, x)=\frac{n}{3 \pi} \int_{-3 \pi / 2 n}^{3 \pi / 2 n} S_{n}(f, x-t) d t
$$

для некоторых $f \in C_{2 \pi}$ будут неограниченно расходится.

А.. Тиман [6] рассматривал средние

$$
\frac{1}{2}\left[S_{n}(f, x)+S_{n}\left(f, x+2 \pi / P_{n}\right)\right]
$$

и показал, что они равномерно сходятся к $f(x)$ для всех $f \in C_{2 \pi}$ тогда и только тогда, когда

$$
\frac{1}{P_{n}}=\frac{k}{2 n+1}+O(1 / n \ln n)
$$

а $k=k(n)$ - целочисленная функция от $n=1,2, \ldots$, принимаюшая лишш конечное число нечетных значений.

Из неравенств (2.40) и (2.28) немедленно следует также 
Teopema 2.5. Ecлu $P_{n}>0, P_{n} \rightarrow+\infty u$

$$
\sup _{n}\left(\frac{P_{n} \ln P_{n}}{n}\left|\sin \frac{\pi n}{P_{n}}\right|\right)<+\infty
$$

то для любой интегрируемой на $[-\pi, \pi]$ периодической с периодом $2 \pi$ функции $f(x)$ средние (2.43) сходятся $\kappa f(x)$ в точках Лебега этой функции.

ДоКАЗАТЕЛЬСТво. Из (2.51) следует

$$
\frac{P_{n}}{n}\left|\sin \frac{\pi n}{P_{n}}\right|<\frac{c_{\left\{P_{n}\right\}}}{\ln P_{n}},
$$

где $c_{\left\{P_{n}\right\}}$ - постоянная, зависящая от последовательности $\left\{P_{n}\right\}$.

Поэтому из (2.40) следует неравенство

$$
\left|F_{n, P_{n}}(x)\right| \leqslant \frac{c_{1}}{n}+\frac{c_{2}}{P_{n} x^{2}}+\frac{c^{(1)}\left(\left\{P_{n}\right\}\right)}{\ln P_{n}}+\frac{c^{(2)}\left(\left\{P_{n}\right\}\right)}{|x| \ln P_{n}}, \quad|x| \geqslant \frac{2 \pi}{P_{n}},
$$

где $c_{1}, c_{2}$ - абсолютные постоянные, а $c^{(1)}\left(\left\{P_{n}\right\}\right), c^{(2)}\left(\left\{P_{n}\right\}\right)$ - постоянные, зависящие от последовательности $\left\{P_{n}\right\}$.

Обозначим

$$
\Gamma_{n, P_{n}}(x)=\min \left\{\frac{2 P_{n}}{\pi},\left(\frac{c_{1}}{n}+\frac{c_{2}}{P_{n} x^{2}}+\frac{c^{(1)}\left(\left\{P_{n}\right\}\right)}{\ln P_{n}}+\frac{c^{(2)}\left(\left\{P_{n}\right\}\right)}{|x| \ln P_{n}}\right)\right\}, \quad|x| \leqslant \pi .
$$

Очевидно, $\Gamma_{n, P_{n}}(x)$ возрастает на $[-\pi, 0]$ и убывает на $[0, \pi]$ и при этом согласно $(2.53)$ и $(2.28)$ имеем

$$
\left|F_{n, P_{n}}(x)\right| \leqslant \Gamma_{n, P_{n}}(x) .
$$

Очевидно также, что

$$
\Gamma_{n, P_{n}}(x) \geqslant 0, \quad \int_{-\pi}^{\pi} \Gamma_{n, P_{n}}(x) d x \leqslant c\left(\left\{P_{n}\right\}\right),
$$

где $c\left(\left\{P_{n}\right\}\right)$ - постоянная, зависяшая от последовательности $\left\{P_{n}\right\}$. Кроме того, из $(2.54)$ следует, что для любого $\delta>0$

$$
\lim _{n \rightarrow \infty} \max _{|x|>\delta} \Gamma_{n, P_{n}}(x)=0 .
$$

Так как (см. (2.16))

$$
\Phi_{n, P_{n}}(f, x)=\frac{P_{n}}{2 \pi} \int_{-\pi / P_{n}}^{\pi / P_{n}} S_{n}(f, x-t) d t=\int_{-\pi}^{\pi} f(x+t) F_{n, P_{n}}(t) d t,
$$

то из (2.55)-(2.58) и (2.23) следует, что можно применить теорему Д.К. Фадеева (см. [7] и [8, с. 265]). Согласно этой теореме средние (2.58) при $n \rightarrow \infty$ будут сходиться к $f(x)$ во всех точках $x$, являюшимися точками Лебега функции $f$. Теорема 2.5 доказана.

Аналогичньм методом доказьвается, что верна 
Teopema 2.6. Среднuе

$$
\frac{a_{0}}{2}+\sum_{k=1}^{n}\left(\frac{\sin k \pi / P_{n}}{k \pi / P_{n}}\right)^{2}\left(a_{k} \cos k x+b_{k} \sin k x\right),
$$

где $a_{k}, b_{k}-$ коэффициенты Фурье функции $f(x)$, сходятся равномерно $\kappa f(x)$ для всех $f \in C_{2 \pi}$ тогда и только тогда, когда

$$
\sup _{n}\left(\frac{P_{n}^{2} \ln P_{n}}{n^{2}} \sin ^{2} \frac{\pi n}{P_{n}}\right)<+\infty
$$

и при выполнении (2.60) средние (2.59) для любой интегрируемой $2 \pi$-периодической функиии $f(x)$ сходятся $\kappa f(x)$ во всех ее точках Лебега.

ЗамечаниЕ. При $P_{n}=n$ имеем

$$
\begin{gathered}
\frac{a_{0}}{2}+\sum_{k=1}^{n}\left(\frac{\sin k \pi / n}{k \pi / n}\right)\left(a_{k} \cos k x+b_{k} \sin k x\right)=\frac{n}{2 \pi} \int_{x-\pi / n}^{x+\pi / n} S_{n}(f, x-t) d t \\
\frac{a_{0}}{2}+\sum_{k=1}^{n}\left(\frac{\sin k \pi / n}{k \pi / n}\right)^{2}\left(a_{k} \cos k x+b_{k} \sin k x\right)=\int_{x-2 \pi / n}^{x+2 \pi / n} S_{n}(f, x-t) n \Phi(n t) d t
\end{gathered}
$$

где

$$
\Phi(t)= \begin{cases}\frac{1}{2 \pi}\left(1-\frac{|t|}{2 \pi}\right), & |t|<2 \pi, \\ 0, & |t| \geqslant 2 \pi,\end{cases}
$$

и согласно теоремам 2.5 и 2.6 средние (2.61) и (2.62) сходятся к $f(x)$ во всех ее точках Лебега. С другой стороны, согласно теореме А.Н. Колмогорова (см. [5, т. I]) ряд Фурье интегрируемой функции может почти всюду неограниченно расходиться. Сходимость же почти всюду средних (2.61) и (2.62) такого ряда означает, что в интервалах типа $\left(x_{0}-\pi / n, x_{0}+\pi / n\right),\left(x_{0}-2 \pi / n, x_{0}+2 \pi / n\right)$ для почти всех $x_{0}$ происходит интерференция значений частных сумм $S_{n}(f, x)$ отклоняющихся от значения $f\left(x_{0}\right)$ в большую и меньшую стороны. Любопытно, что это явление не имеет места в интервалах вида, например, $\left(x_{0}-\pi / 3 n, x_{0}+\pi / 3 n\right),\left(x_{0}-2 \pi / 3 n, x_{0}+2 \pi / 3 n\right)$.

Точнее, имеет место следующая

Tеорема 2.7. Если число $h>0$ не целое и $n / P_{n}=h, n=1,2, \ldots$, mo существует интегрируемая на $[0,2 \pi]$ функиия $f(x)$ такая, что средние (2.43) и (2.59) расходятся почти всюду.

Это утверждение можно доказать дословньм повторением рассуждений доказательства теоремы А.Н. Колмогорова о существовании интегрируемой функции с расходящимся почти всюду рядом Фурье.

ЗАмечАниЕ. В теоремах 2.5 и 2.6 матрицы $\lambda_{k}^{(n)}, 1 \leqslant k \leqslant n$, средние (2.23) и (2.59), вообще говоря, не обладают свойством вьпуклости вышеприведенной теоремы С. М. Никольского, и поэтому для их доказательства понадобилось отдельное изучение соответствуюших ядер. 


\section{§ 3. О сходимости почти всюду средних Римана и Стеклова частных сумм кратных рядов Фурье}

Пусть $\mathbb{R}^{q}$ - пространство векторов $\mathbf{x}=\left(x_{1}, \ldots, x_{q}\right), \mathbf{t}=\left(t_{1}, \ldots, t_{q}\right)$ с вещественными координатами,

$$
\alpha \mathbf{x}+\beta \mathbf{t}=\sum_{j=1}^{q} \alpha x_{j}+\beta t_{j}, \quad \mathbf{x} \cdot \mathbf{t}=\sum_{j=1}^{q} x_{j} t_{j}
$$

$\mathbf{T}_{q}=[-\pi, \pi)^{q}$, а $C\left(\mathbf{T}_{q}\right)$ есть множество непрерывных на $\mathbb{R}^{q}$ периодических, с периодом $2 \pi$ по каждой переменной, функций $f(\mathbf{x}), L_{1}\left(\mathbf{T}_{q}\right)$ - множество интегрируемых на $\mathbf{T}_{q}$ периодических функций, $L\left(1+\log ^{m-1} L\right)\left(\mathbf{T}_{q}\right)$ - множество тех функций $f(\mathbf{x}) \in L\left(\mathbf{T}_{q}\right)$, для которых конечен интеграл

$$
\begin{aligned}
\int_{\mathbf{T}_{q}} & |f(\mathbf{x})|\left[1+\left(\log ^{+}|f(\mathbf{x})|\right)^{q-1}\right] d \mathbf{x} \\
\quad & =\int_{-\pi}^{\pi} \cdots \int_{-\pi}^{\pi}\left|f\left(x_{1}, \ldots, x_{q}\right)\right|\left[1+\left(\log ^{+}\left|f\left(x_{1}, \ldots, x_{q}\right)\right|\right)^{q-1}\right] d x_{1} \cdots d x_{q} .
\end{aligned}
$$

Определяемая вектором $\mathbf{n}=\left(n_{1}, \ldots, n_{q}\right)$, где $n_{j}$ - натуральные числа, прямоугольная частная сумма ряда Фурье функции $f\left(x_{1}, \ldots, x_{q}\right)$ обозначается через $S_{\mathbf{n}}(f, \mathbf{x})$ или $S_{n_{1}, \ldots, n_{q}}\left(f, x_{1}, \ldots, x_{q}\right)$, т.е. полагается

$$
S_{\mathbf{n}}(f, \mathbf{x})=\sum_{|\mathbf{k}| \leqslant \mathbf{n}} a_{\mathbf{k}} e^{i \mathbf{k x}}=\sum_{k_{1}=-n_{1}}^{n_{1}} \cdots \sum_{k_{q}=-n_{q}}^{n_{q}} a_{k_{1} \ldots k_{q}} e^{i\left(k_{1} x_{1}+\cdots+k_{q} x_{q}\right)},
$$

где

$$
\begin{aligned}
a_{\mathbf{k}}=a_{k_{1} \ldots k_{q}} & =\frac{1}{(2 \pi)^{q}} \int_{\mathbf{T}_{q}} f(\mathbf{x}) e^{-i \mathbf{n} \mathbf{x}} d \mathbf{x} \\
& =\frac{1}{(2 \pi)^{q}} \int_{-\pi}^{\pi} \cdots \int_{-\pi}^{\pi} f\left(x_{1}, \ldots, x_{q}\right) e^{-i\left(n_{1} x_{1}+\cdots+n_{q} x_{q}\right)} d x_{1} \cdots d x_{q} .
\end{aligned}
$$

Пусть $\left\{P_{n_{j}}^{(j)}\right\}_{n_{j}=1}^{\infty}, 1 \leqslant j \leqslant q,-$ последовательности положительных чисел, каждая из которых монотонно стремится к $+\infty$ при $n_{j} \rightarrow+\infty$. Обозначим $P_{\mathbf{n}}=$ $\left(P_{n_{1}}^{(1)}, \ldots, P_{n_{q}}^{(q)}\right)$, где $n=\left(n_{1}, \ldots, n_{q}\right)$, и положим

$$
\begin{gathered}
Q_{\pi, P_{\mathbf{n}}}^{(m)}(\mathbf{t})=\prod_{j=1}^{q} P_{n_{j}}^{(j)} \Phi_{\pi}^{(m)}\left(P_{n_{j}}^{(j)} t_{j}\right) \\
\Phi_{\pi, P_{\mathbf{n}}^{(m)}}(f, \mathbf{x})=\int_{\mathbb{R}^{q}} S_{\mathbf{n}}(f, \mathbf{x}-\mathbf{t}) Q_{\pi, P_{\mathbf{n}}}(\mathbf{t}) d \mathbf{t},
\end{gathered}
$$

где

$$
\begin{aligned}
\Phi_{\pi}^{(1)} & = \begin{cases}1 / 2 \pi, & x \in[-\pi, \pi], \\
0, & x \notin[-\pi, \pi],\end{cases} \\
\Phi_{\pi}^{(m)}(x) & =\int_{\mathbb{R}_{q}} \Phi_{\pi}^{(m-1)}(x-t) \Phi_{\pi}^{(1)} d t .
\end{aligned}
$$


Обозначим через $F_{\mathbf{n}, P_{\mathbf{n}}}^{(m)}(\mathbf{x})$ ядро преобразования $(3.4)$, т.е

$$
F_{\mathbf{n}, P_{\mathbf{n}}}^{(m)}(\mathbf{x})=\frac{1}{\pi^{q}} \int_{\mathbb{R}^{q}} D_{\mathbf{n}}(\mathbf{x}-\mathbf{t}) Q_{\pi, P_{\mathbf{n}}}^{(m)}(\mathbf{t}) d \mathbf{t}
$$

где

$$
D_{\mathbf{n}}(\mathbf{x})=\prod_{j=1}^{q} D_{n_{j}}\left(x_{j}\right)
$$

Очевидно,

$$
F_{\mathbf{n}, P_{\mathbf{n}}}^{(m)}(\mathbf{x})=\prod_{j=1}^{q} F_{n_{j}, P_{n_{j}}^{(j)}}^{(m)}\left(x_{j}\right)
$$

где

$$
F_{n_{j}, P_{n_{j}}^{(j)}}^{(m)}\left(x_{j}\right)=\frac{1}{\pi} \int_{\mathbb{R}_{1}} D_{n_{j}}\left(x_{j}-t_{j}\right) P_{n_{j}}^{(j)} \Phi_{\pi}\left(P_{n_{j}}^{(j)} t_{j}\right) d t_{j} .
$$

Легко видеть также, что

$$
\begin{aligned}
& \Phi \\
& =\int_{\mathbf{T}_{q}, P_{\mathbf{n}}^{(m)}} f(\mathbf{t}) F_{\mathbf{n}, P_{\mathbf{n}}}^{(m)}(\mathbf{x}-\mathbf{t}) d \mathbf{t}=\int_{\mathbf{T}_{q}} f(\mathbf{x}-\mathbf{t}) F_{\mathbf{n}, P_{\mathbf{n}}}^{(m)}(\mathbf{t}) d \mathbf{t} \\
& =\sum_{k_{1}=-n_{1}}^{n_{1}} \ldots \sum_{k_{q}=-n_{q}}^{n_{q}} a_{k_{1} \ldots k_{q}}\left(\frac{\sin k_{1} \frac{\pi}{P_{n_{1}}^{(1)}}}{k_{1} \frac{\pi}{P_{n_{1}}^{(1)}}}\right)^{m} \cdots\left(\frac{\sin k_{q} \frac{\pi}{P_{n_{q}}^{(q)}}}{k_{q} \frac{\pi}{P_{n_{q}}^{(q)}}}\right)^{m} e^{i k_{1} x_{1} \ldots e^{i k_{q} x_{q}} .}
\end{aligned}
$$

В предыдущем параграфе были найдены необходимые и достаточные условия на последовательности $P_{n_{j}}^{(j)}, n_{j}=1,2, \ldots$, при выполнении которых нормы в $L_{1}$ ядер $F_{n_{j}, P_{n_{j}}^{(j)}}^{(m)}\left(x_{j}\right)$ ограничены (см. теоремы $2.2,2.3,2.4$ и 2.6$)$. В силу $(3.9)$ эти условия приводят к необходимьм и достаточным условиям на векторы $P_{\mathbf{n}}=\left(P_{n_{1}}^{(1)}, \ldots, P_{n_{q}}^{(q)}\right)$, при выполнении которых для всех $\mathbf{n}=\left(n_{1}, \ldots, n_{q}\right)$

$$
\int_{\mathbf{T}_{q}}\left|F_{\mathbf{n}, P_{\mathbf{n}}}\right| d \mathbf{x} \leqslant M(m), \quad m \geqslant 1
$$

Условимся писать $\mathbf{n}=\left(n_{1}, \ldots, n_{m}\right) \rightarrow \infty$, если все координаты $n_{j}$ независимо друг от друга стремятся к $\infty$.

Из указанных теорем 2.2, 2.3, 2.4, и 2.6 следует

ТЕОрема 3.1. Для того чтобъ средние $\Phi_{\pi, P_{\mathbf{n}}}(f, \mathbf{x}), m \geqslant 3$, nри $\mathbf{n} \rightarrow \infty$ равномерно сходились $\kappa f(\mathbf{x})$ для всех $f(x) \in C\left(\mathbf{T}_{q}\right)$, необходимо и достаточно выполнение неравенств

$$
\sup _{n_{j}}\left(\frac{P_{n_{j}}}{n_{j}}\right)^{(j)}\left|\sin \frac{\pi n_{j}}{P_{n_{j}}^{(j)}}\right|^{m}\left|\ln n_{j}\right|<+\infty, \quad 1 \leqslant j \leqslant q .
$$


Для равномерной сходимости средних $\Phi_{\pi}^{(m)}(f, \mathbf{x})$ nри $m=1$ и $m=2$ на всем классе $f \in C\left(\mathbf{T}_{q}\right)$ необходимо и достаточно соответственно выполнение неравенств

$$
\begin{gathered}
\sup _{n_{j}}\left(\frac{P_{n_{j}}{ }^{(j)}}{n_{j}}\right)\left|\sin \frac{\pi n_{j}}{P_{n_{j}}^{(j)}}\right|\left|\ln P_{n_{j}}\right|<+\infty, \quad 1 \leqslant j \leqslant q, \\
\sup _{n_{j}}\left(\frac{P_{n_{j}}^{(j)}}{n_{j}}\right)^{2}\left|\sin ^{2} \frac{\pi n_{j}}{P_{n_{j}}^{(j)}}\right|\left|\ln P_{n_{j}}\right|<+\infty, \quad 1 \leqslant j \leqslant q .
\end{gathered}
$$

ЗАмечАнИЕ. При $P_{n_{j}}^{(j)}=n_{j}, 1 \leqslant j \leqslant q$, условия (3.12)-(3.14) выполнены. Поэтому из теоремы 3.1 и из равенства (3.10) следует

ТЕОрема 3.2. Для любого $m \geqslant 1 u f(\mathbf{x}) \in C\left(\mathbf{T}_{q}\right)$ полиномы

$$
\begin{aligned}
\Phi_{\pi, \mathbf{n}}^{(m)}(f, \mathbf{x})= & \sum_{k_{1}=-n_{1}}^{n_{1}} \ldots \sum_{k_{q}=-n_{q}}^{n_{q}} a_{k_{1} \ldots k_{q}} \\
& \times\left(\frac{\sin k_{1} \pi / n_{1}}{k_{1} \pi / n_{1}}\right)^{m} \cdots\left(\frac{\sin k_{q} \pi / n_{q}}{k_{q} \pi / n_{q}}\right)^{m} e^{i k_{1} x_{1}} \ldots e^{i k_{q} x_{q}},
\end{aligned}
$$

где $a_{k_{1} \ldots k_{q}}$ - коэффичиенты Фурье функиии $f(\mathbf{x})$, равномерно на $\mathbb{R}^{q}$ сходятся $\kappa f(\mathbf{x})$ nрu $\mathbf{n} \rightarrow \infty$.

Как видно из вьшеизложенных результатов, средние $\Phi_{\pi, \mathbf{n}}^{(m)}(f, \mathbf{x})$ при вьполнении условий (3.12)-(3.14) как в одномерном, так и в многомерном случае обладают свойствами, близкими к свойствам чезаровских и абелевских средних. Следующая теорема представляет аналог известной теоремы о $(C, 1)$-средних (см. [5, т. 2, с. 463]).

Теорема 3.3. Если векторы $P_{\mathbf{n}}=\left(P_{n_{1}}^{(1)}, \ldots, P_{n_{q}}^{(q)}\right)$ mаковы, что $P_{n_{j}}^{(j)}>0$, $n_{j}=1,2, \ldots, P_{n_{j}} \uparrow+\infty$ nри $n_{j} \rightarrow+\infty, 1 \leqslant j \leqslant q$, и выполнены неравенства (3.13), то для любой функции $f(\mathbf{x}) \in L\left(\mathbf{T}_{q}\right)$, для которой на $\mathbf{T}_{q}$ интегрируема также функиия $|f(\mathbf{x})| \ln ^{+}|f(\mathbf{x})|^{q-1}$, средние $\Phi_{\pi, \mathbf{n}}^{(1)}(f, \mathbf{x})$ при $\mathbf{n} \rightarrow \infty$ сходятся $\kappa f(\mathbf{x})$ почти всюду на $\mathbf{T}_{q}$.

Ниже мы проводим доказательство одной обшей теоремы, из которой в частности вытекает также теорема 3.3.

ТЕОРемА 3.4. Пусть функиии $\left\{\varphi_{n_{j}}^{(j)}\left(x_{j}\right)\right\}_{n_{j}=1}^{\infty}, 1 \leqslant j \leqslant q$, удовлетворяют следующим условиям.

1) Существуют определенные на $[-\pi, \pi]$ неотрицательные ограниченные четные и монотонно убывающие на $[0, \pi]$ функиии $\left\{\Psi_{n_{j}}^{(j)}\left(x_{j}\right)\right\}_{n_{j}=1}^{\infty}, 1 \leqslant j \leqslant q$, такие, что

$$
\begin{gathered}
\varphi_{n_{j}}^{(j)}\left(x_{j}\right) \leqslant \psi_{n_{j}}^{(j)}\left(x_{j}\right), \quad x_{j} \in[-\pi, \pi], \quad n_{j}=1,2, \ldots, \quad 1 \leqslant j \leqslant q, \\
\sup _{n_{j}} \int_{-\pi}^{\pi} \psi_{n_{j}}^{(j)}\left(x_{j}\right) d x_{j}<+\infty, \quad 1 \leqslant j \leqslant q, \\
\lim _{n_{j} \rightarrow \infty} \Psi_{n_{j}}^{(j)}\left(x_{j}\right)=0, \quad x_{j} \neq 0, \quad 1 \leqslant j \leqslant q .
\end{gathered}
$$


2) Для всех $j, 1 \leqslant j \leqslant q$, выполняются равенства

$$
\lim _{n_{j} \rightarrow \infty} \int_{-\pi}^{\pi} \varphi_{n_{j}}\left(x_{j}\right) d x_{j}=1 .
$$

Тогда для любой функции $f(\mathbf{x}) \in L\left(\mathbf{T}_{q}\right)$, для которой на $\mathbf{T}_{q}$ интегрируема также функиия $|f(b f x)|\left(\ln ^{+}|f(\mathbf{x})|^{q-1}\right)$, почти всюду на $\mathbf{T}_{q}$ имеет место равенство

$$
\lim _{\mathbf{n} \rightarrow \infty} \int_{\mathbf{T}_{q}} f(\mathbf{x}-\mathbf{t}) Q_{\mathbf{n}}(\mathbf{t}) d \mathbf{t}=f(\mathbf{x}),
$$

2дe

$$
Q_{\mathbf{n}}(\mathbf{t})=\prod_{j=1}^{q} \varphi_{n_{j}}^{(j)}\left(t_{j}\right), \quad n=\left(n_{1}, \ldots, n_{q}\right), \quad \mathbf{t}=\left(t_{1}, \ldots, t_{q}\right)
$$

Из доказательства теоремы 2.5 видно, что ядра $F_{n_{j}, P_{n_{j}}^{(j)}}^{(1)}\left(x_{j}\right), 1 \leqslant j \leqslant n$, при вьполнении условий (3.13) удовлетворяют всем условиям сформулированной теоремы 3.4 (см. (2.23), (2.56)-(2.58)). Поэтому если положить в теореме 3.4

$$
\varphi_{n_{j}}^{(j)}\left(x_{j}\right)=F_{n_{j}, P_{j}^{(j)}}^{(1)}\left(x_{j}\right), \quad \psi_{n_{j}}^{(j)}\left(x_{j}\right)=\Gamma_{n_{j}, P_{j}^{(j)}}^{(1)}\left(x_{j}\right), \quad 1 \leqslant j \leqslant q,
$$

получим теорему 3.3 .

Точно таким же образом можно убедится, что при вьполнении условий (3.14) утверждение теоремы 3.3 верно для средних $\Phi_{\pi, P_{\mathbf{n}}}^{(2)}(f, \mathbf{x})$.

ДОКАЗАТЕЛЬСТВО ТЕОРЕМЫ 3.4 в некоторой степени аналогично доказательству подобных теорем для кратных средних Чезаро (см. [10], [11]). Покажем, что для любой функции $f \in L^{1}\left(\mathbf{T}_{q}\right)$ выполняется неравенство

$$
\sup _{\mathbf{n}}\left|\int_{\mathbf{T}_{q}} f(\mathbf{x}-\mathbf{t}) Q_{\mathbf{n}}(\mathbf{t}) d t\right| \leqslant c M(f, \mathbf{x})
$$

где $c>0$ - некоторая постоянная, а $M(f, \mathbf{x})$ есть максимальная функция, определенная равенством

$$
M(f, \mathbf{x})=\sup _{t_{1}>0, \ldots, t_{q}>0} \int_{-t_{1}}^{t_{1}} \ldots \int_{-t_{q}}^{t_{q}}|f(\mathbf{x}-\mathbf{t})| d t .
$$

Из (3.21) и (3.16) имеем

$$
Q_{\mathbf{n}}(\mathbf{t}) \leqslant \prod_{j=1}^{q} \Psi_{n_{j}}^{(j)}\left(\left|t_{j}\right|\right)
$$

Легко убедиться, что для любой функции $\Psi_{n_{j}}^{(j)}(t)$ существует кусочно-постоянная функция $\widetilde{\Psi}_{n_{j}}^{(j)}(t)$, монотонно убываюшая на $[0, \pi]$, так, что почти всюду на $[0, \pi]$ вьполняется неравенство

$$
\Psi_{n_{j}}^{(j)}(t) \leqslant \widetilde{\Psi}_{n_{j}}^{(j)}(t) \leqslant 2 \Psi_{n_{j}}^{(j)}(t) .
$$


Далее очевидно, что каждую функцию $\widetilde{\Psi}_{n_{j}}^{(j)}(|t|)$, определенную на $[-\pi, \pi]$, можно представить в виде конечной линейней комбинации функций вида $1 / 2 h \chi_{[-h, h]}$. Причем все коэффициенты этой комбинации положительны и их сумма ограничена некоторьм числом $c>0$. Из последнего с учетом (3.24) следует, что

$$
\left|Q_{\mathbf{n}}(\mathbf{t})\right| \leqslant \sum \alpha_{j} \frac{1}{\left|I_{j}\right|} \chi_{I_{j}}(\mathbf{t})
$$

для каких-то параллелепипедов $I_{j} \subset \mathbb{R}^{n}$ с центром 0, где $\alpha_{j}>0, \sum \alpha_{j}<c$. Отсюда вытекает неравенство

$$
\left|\int_{\mathbb{R}^{n}} f(\mathbf{x}-\mathbf{t}) Q_{\mathbf{n}}(\mathbf{t}) d t\right| \leqslant \sum \alpha_{j} \frac{1}{\left|I_{j}\right|} \int_{I_{j}}|f(\mathbf{x}-\mathbf{t})| d t \leqslant M(f, \mathbf{x}),
$$

которое дает нам (3.23). Для непрерывных функций $g \in C\left(\mathbf{T}_{q}\right)$ из (3.16)-(3.19) очевидно следует равномерная сходимость в (3.20). Далее, как обычно делается, надо приблизить $f$ непрерывной функцией $g \in C\left(\mathbf{T}_{q}\right)$ и применить неравенство типа $(1,1)$ для максимальной функции $M(f, \mathbf{x})($ см. [12, с. 32]). Теорема 3.4 доказана.

\section{Список литературы}

1. Никольский C. М. О линейных методах суммирования рядов Фурье // Изв. АН СССР. Сер. матем. 1948. Т. 12. № 3. С. 259-278.

2. Nagy B. Methodes de sommation des series de Fourier. III // Acta Sci. Math. (Szeged). V. 13. P. $247-351$.

3. Теляковский C. А. О приближении дифференцируемых функций линейными средними их рядов Фурье // Изв. АН СССР. Сер. матем. 1960. Т. 24. № 2. С. 213-242.

4. Тригуб Р. М. Связь суммируемостью и абсолютной сходимостью рядов и преобразований Фурье // Докл. АН СССР. 1974. Т. 217. № 1. С. 34-37.

5. Зигмунд А. Тригонометрические ряды. Т. I, II. М.: Мир, 1965.

6. Тиман A. Ф. О некоторых методах суммирования рядов Фурье // Изв. АН СССР. Сер. матем. 1950. Т. 14. С. 85-94.

7. Фадеев Д. К. О представлении суммируемых функций сингулярными интегралами в точках Лебега // Матем. сб. 1936. Т. 1 (43). №3. С. 351-368.

8. Натансон И. П. Теория функций вещественной переменной. М.: Наука, 1974.

9. Стечкин С. Б. Методы суммирования С.Н. Бернштейна и В. Рогозинского. Г. Харди. Расходящиеся ряды (обзорная статья редактора). М.: ИЛ, 1951.

10. Jessen B., Marcinkiewicz J., Zygmund A. Note one differentiability of multiple integrals // Fund. Math. 1935. V. 25. P. 217-234.

11. Marcinkiewicz J., Zygmund A. On summability of double Fourier series // Fund. Math. 1939. V. 32. P. 112-132.

12. Гусман М. Дифференцирование интегралов в $\mathbb{R}^{n}$. М.: Мир, 1977.

13. Nagy B. Sur une class generale de procedes de sommation pour les series de Fourier // Acta. Math. Hungar. 1948. V. 1. № 3. P. 14-52.

14. Ефимов А. В. О линейных методах суммирования рядов фурье // Изв. АН СССР. Сер. матем. 1960. Т. 24. С. 743-756.

15. Теляковский C.A. О нормах тригонометрических полиномов и приближении дифференцируемых функций линейньми средними их рядов Фурье. I // Труды МИАН. 1961. T. 62. C. 61-97. 